\title{
Interplanetary Trajectory Design for the Asteroid Robotic Redirect Mission Alternate Approach Trade Study
}

\author{
Raymond Gabriel Merrill ${ }^{1}$ \\ NASA Langley Research Center, Hampton, VA., 23681, USA \\ $\mathrm{Min} \mathrm{Qu}^{2}$ \\ Analytic Mechanics Associates, Hampton, VA., 23666, USA \\ Matthew A. Vavrina ${ }^{3}$ \\ a.i. solutions, Inc., Lanham, MD 20706, USA \\ Jacob A. Englander ${ }^{4}$ \\ NASA Goddard Spaceflight Center, Greenbelt, MD, 20771, USA \\ and \\ Christopher A. Jones 5 \\ NASA Langley Research Center, Hampton, VA., 23681, USA
}

\begin{abstract}
This paper presents mission performance analysis methods and results for the Asteroid Robotic Redirect Mission (ARRM) option to capture a free standing boulder on the surface of a $100 \mathrm{~m}$ or larger NEA. It details the optimization and design of heliocentric low-thrust trajectories to asteroid targets for the ARRM solar electric propulsion spacecraft. Extensive searches were conducted to determine asteroid targets with large pick-up mass potential and potential observation opportunities. Interplanetary trajectory approximations were developed in method based tools for Itokawa, Bennu, $1999 \mathrm{JU}_{3}$, and $2008 \mathrm{EV}_{5}$ and were validated by end-to-end integrated trajectories.
\end{abstract}

\section{Introduction}

A key component of NASA's asteroid initiative is the Asteroid Robotic Redirect Mission (ARRM). Initially NASA requested a study of the feasibility of a mission to capture an entire four to ten meter diameter asteroid and redirect it to trans-lunar space ${ }^{2}$ (Option A). An alternate ARRM concept (Option B) also being studied by NASA in the Alternate Approach Trade Study (AATS) is to capture a two to four meter boulder from the surface of a larger $100+$ meter diameter Near Earth Asteroid (NEA) and redirect it to trans-lunar space. ${ }^{3}$ Option B provides NASA the option to trade potential return mass for centimeter level characterization of an entire large NEA, greater certainty of target NEA composition, ability to select the boulder captured, additional experience operating in a micro gravity environment including extended surface contact, and the ability to perform one or more Planetary Defense (PD) demonstrations on a large NEA. In the ARRM Alternate Concept Study the Option B concept was investigated to determine feasibility and identify potential differences from the ARRM Option A. Mission design

${ }^{1}$ Aerospace Engineer, Space Mission Analysis Branch, NASA Langley Research Center, Hampton, VA, 23681, USA, Senior Member AIAA

${ }^{2}$ Staff Scientist, Analytical Mechanics Associates, Inc., 21 Enterprise Parkway, Suite 300, Hampton, VA 23666, USA

${ }^{3}$ Senior Systems Engineer, a.i. solutions, Inc., 10001 Derekwood Ln. Suite 215, Lantham, MD, 20706, USA

${ }^{4}$ Aerospace Engineer, Navigation and Mission Design Branch, NASA Goddard Space Flight Center, Greenbelt, MD, 20771, USA, Member AIAA

${ }^{5}$ Aerospace Engineer, Space Mission Analysis Branch, NASA Langley Research Center, Hampton, VA, 23681, USA, Member AIAA 
for Option B includes many similarities with Option A including total propellant carried, Earth departure, Earth arrival, and trans-lunar parking options accessible to crewed visits via the Space Launch System (SLS). It also differs significantly; there are three NEAs (Itokawa, Bennu, $1999 \mathrm{JU}_{3}$ ) that have past or planned precursor missions that are potential targets. Additionally, radar observation of $2008 \mathrm{EV}_{5}$ has identified potential boulder reflections, and surface roughness indicates the presence of many boulders in the two to four meter range. ${ }^{4,5}$ Option B also differs in the distance from the Earth and Sun achieved, in many cases out to Mars-like distances. Other key difference include the time for proximity operations, thrust-to-weight of the vehicle after boulder collection, and robustness of individual NEA targets to launch and return date slips. Option B returns a boulder on the two to four meter diameter scale by leveraging a high-efficiency ( $\mathrm{I}_{\mathrm{sp}}$ : $\left.2000-3000 \mathrm{~s}\right)$, high-power $(40-50 \mathrm{~kW})$ Solar Electric Propulsion (SEP) system in development by NASA's Space Technology Mission Directorate (STMD).

A critical aspect of Option B is the development of interplanetary trajectory options that identify the size of the boulder that can be returned from candidate NEAs. The trajectory design for Option B is complex for a variety of reasons. While the SEP system offers the propellant efficiency required to return a massive boulder from an asteroid to a trans-lunar orbit, it is only capable of relatively low-thrust $(<2 \mathrm{~N})$. Thus, long periods of thrust are required to achieve the necessary $\Delta \mathrm{V}$ for a mission, and low-thrust trajectory optimization is necessary to identify feasible trajectories composed of long thrust arcs. Additionally, for any candidate NEA the return mass is subject to the interdependence between mission duration, launch date, launch vehicle capacity, spacecraft dry mass, spacecraft propellant, SEP system $I_{\text {sp }}$, SEP system propellant efficiency, and power available from the solar arrays for thrusting. These system characteristics can be traded within limits to maximize the return mass. Also, unlike Option A, the Option B trajectory pick-up mass at the NEA is not the three sigma upper bound of the entire NEA based on observation, rather it is variable depending on boulder selected. The pick-up mass is maximized in the interplanetary trajectory and used by proximity operations analysts to inform the boulder upper bound that can be captured and redirected.

An initial constraint of a return to the Earth - Moon system by the end of 2024 to enable crewed exploration on an early SLS / Orion mission places an upper bound on the time of flight of the trajectory and eliminates trajectory options that might allow for more optimal phasing with a later return date. The addition of a required stay at the asteroid of over one year for characterization and PD operations also decreases trajectory options due to the reduced time available to thrust. Later return dates, later launch dates, and reduction in stay time have also been assessed and included in the results to illustrate the effect of programmatic decisions and uncertainties on individual target maximum boulder return mass.

Design space complexity is enhanced by the possible inclusion of a gravity assist to reduce $\Delta \mathrm{V}$ requirements. NEA boulder retrieval missions can benefit from energy- and plane-altering flybys of Earth, Mars, or Venus, warranting a thorough search for gravity assists on both the outbound and inbound legs. While gravity assists can improve the maximum boulder pick-up mass, a more expansive design space must be searched. This design space grows significantly when considering target asteroids outside of those that have had or will have precursors before launch. There are over 60 asteroids with a radar observation opportunity before 2020 that have a diameter that is likely greater than $100 \mathrm{~m}$.

The mission design for Option A, to return an entire asteroid, has recently been outlined by several authors. Landau et al. describe trajectories to return all of $2008 \mathrm{HU}_{4}$ and $1998 \mathrm{KY}_{26}$, as well as a massive piece of $2000 \mathrm{SG}_{344}$ using an Atlas V launch vehicle. ${ }^{6}$ Strange et al. extend the concept, examining performance for returning the entire mass of a number of 2 - $26 \mathrm{~m}$ diameter asteroids, including the prime target of 2009 BD for a number of launch vehicles. $^{2}$ Notably, these two studies use lunar gravity assists on Earth-Moon system departure to increase the departure $\mathrm{C} 3$ after a spiral out from a low Earth orbit as described by Landau et al. and McElrath et al. ${ }^{7,8}$ Another series of lunar gravity assists on the return to the Earth-Moon system allows for a decrease in C3 of up to $2 \mathrm{~km} / \mathrm{s}^{2}$ to capture energy with small propellant requirements. Englander and Cardiff shift focus from spiral out trajectories to direct launch options and perform a trade on a variety of thrusters for an ARRM spacecraft. ${ }^{9}$

This paper presents an analysis of mission performance for the ARRM Option B, detailing the optimization and design of low-thrust trajectories to asteroid targets for the ARRM solar electric propulsion (SEP) spacecraft. Both the Evolutionary Mission Trajectory Generator (EMTG) ${ }^{10,11,12,13,14}$ at NASA Goddard Spaceflight Center (GSFC) and the Mission Analysis Low-Thrust Optimization (MALTO) ${ }^{15}$ developed at the NASA Jet Propulsion Laboratory were used to generate interplanetary trajectories to Itokawa, Bennu, $1999 \mathrm{JU}_{3}$, and $2008 \mathrm{EV}_{5}$. Extensive searches were also conducted to determine asteroid targets with large pick-up mass potential. 


\section{Mission Modeling}

Due to the large number of potential targets, a multi-tiered approach was employed to allow a broad look at known NEAs. A high-thrust multi-revolution Lambert solver was first used to scan through all known NEAs and to find mission opportunities between 2010 and 2040. Outbound and inbound trajectories were searched independently for departure windows in every 30 days and flight time in every 30-day increment up to 5 years. Every mission opportunity with a one way $\Delta \mathrm{V}$ requirement of less than $10 \mathrm{~km} / \mathrm{s}$ is recorded in the database for each NEA.

In order to down select the NEAs and allow the ARRM team to focus on NEAs with higher mass return potentials, a ranking formula was developed to rank all NEAs based solely on their orbital elements. The ranking parameter $\mathrm{p}$ is calculated for each NEA based on the delta-V requirement of a 3-burn orbit transfer from the Earth orbit to the NEA orbit, which can be calculated directly using NEA's orbital elements. The first two maneuvers are Hohmann like transfers that change the orbit to match the target orbit's semi-major axis and eccentricity, and the third maneuver changes the orbit inclination at one of NEA orbit's two nodal crossing points, whichever is further away from the Sun. The formula for the ranking parameter $p$ is given by

$$
\mathrm{p}=p 1+p 2+p 9
$$

where

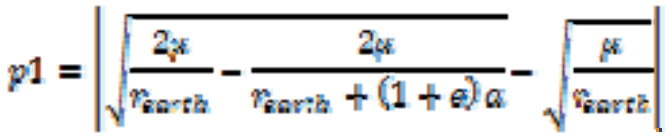

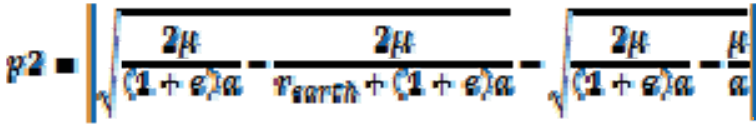

$$
\mu \sigma=L \sqrt{\frac{2 \mu}{r_{\text {crossing }}}-\frac{\mu}{\alpha}}
$$

and

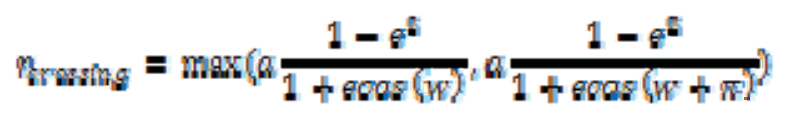

Here $\mu$ is the gravitational constant of the Sun, Parts is radius of the Earth orbit, and $a, e, i, w$ are NEA orbit's semi-major axis, eccentricity, inclination and argument of periapsis respectively.

Figures 1, 2, 3 are the scatter plots of the best Lambert solutions of all NEAs recorded in the database between 2010 and 2040 that show how NEA orbit's semi-major axis, inclination, eccentricity each contribute to the delta-V's individually and Figure 4 shows how the ranking parameters calculated from the 3-burn maneuver sequence compare against the best Lambert solutions. It is clear that even though the 3-burn maneuver sequence does not represent a real mission opportunity since it does not take into consideration the phasing of the orbits, it provides a very good measurement on the potential performance of a NEA in its best mission opportunity. It can also be used to quickly estimate the performance of a newly found NEA based on its orbital elements without having to run a Lambert scan on the target. 


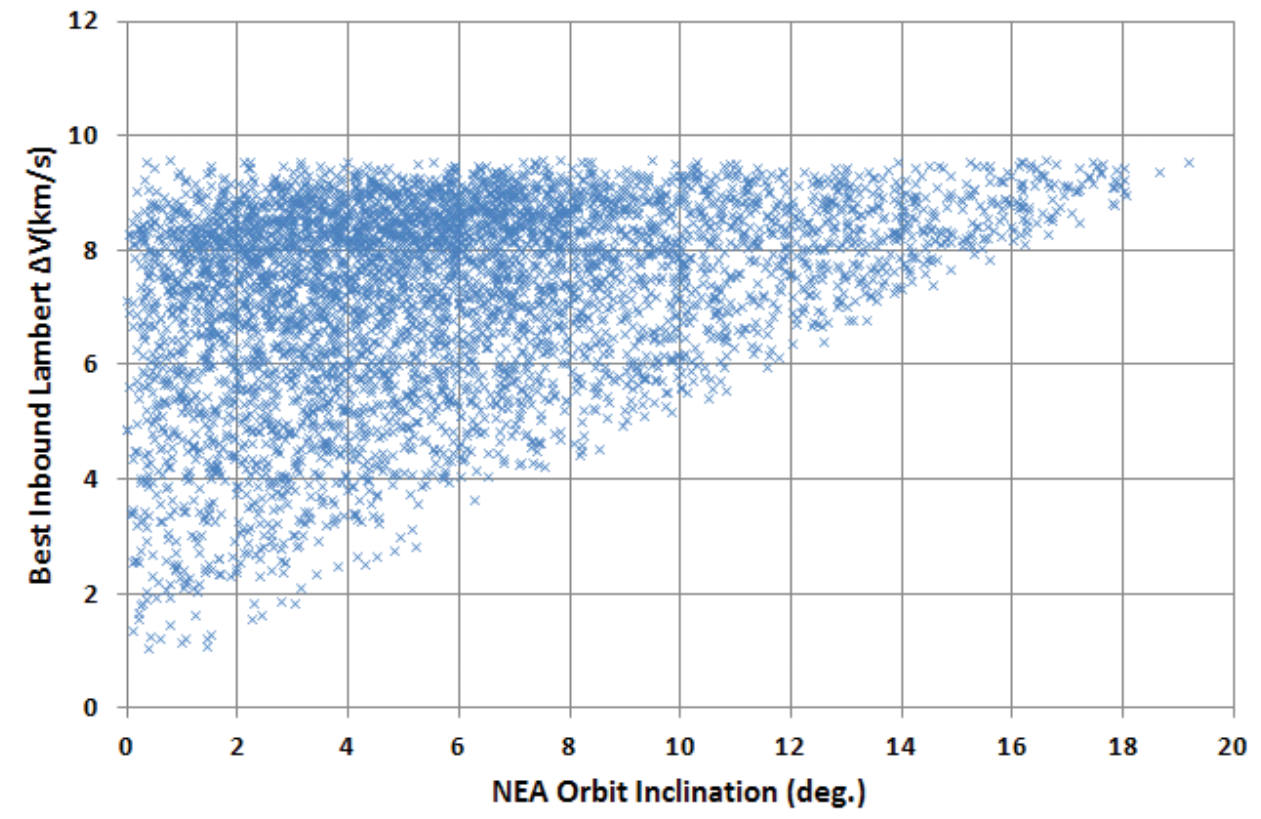

Figure 1. Inclination vs best inbound Lambert $\Delta V$ between 2010 and 2040 for all NEAs recorded

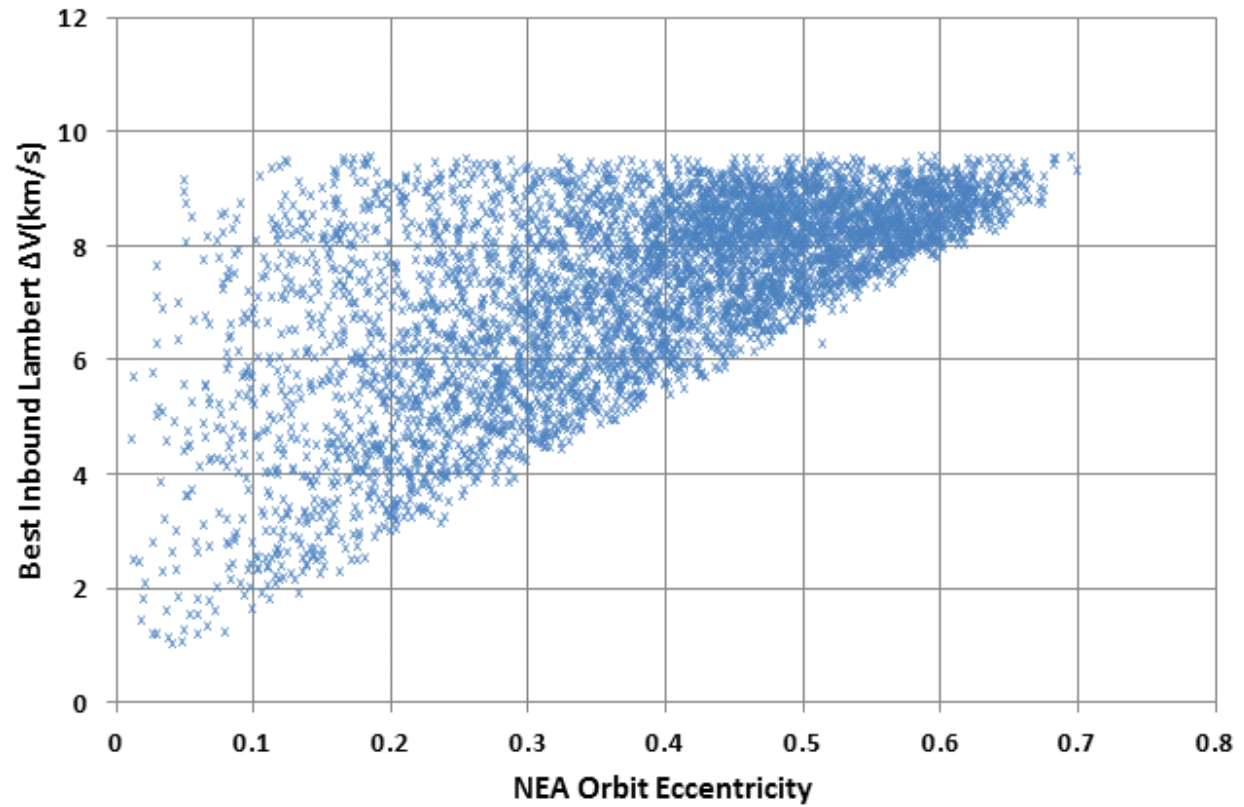

Figure 2. Eccentricity vs best inbound Lambert $\Delta V$ between 2010 and 2040 for all NEAs recorded 


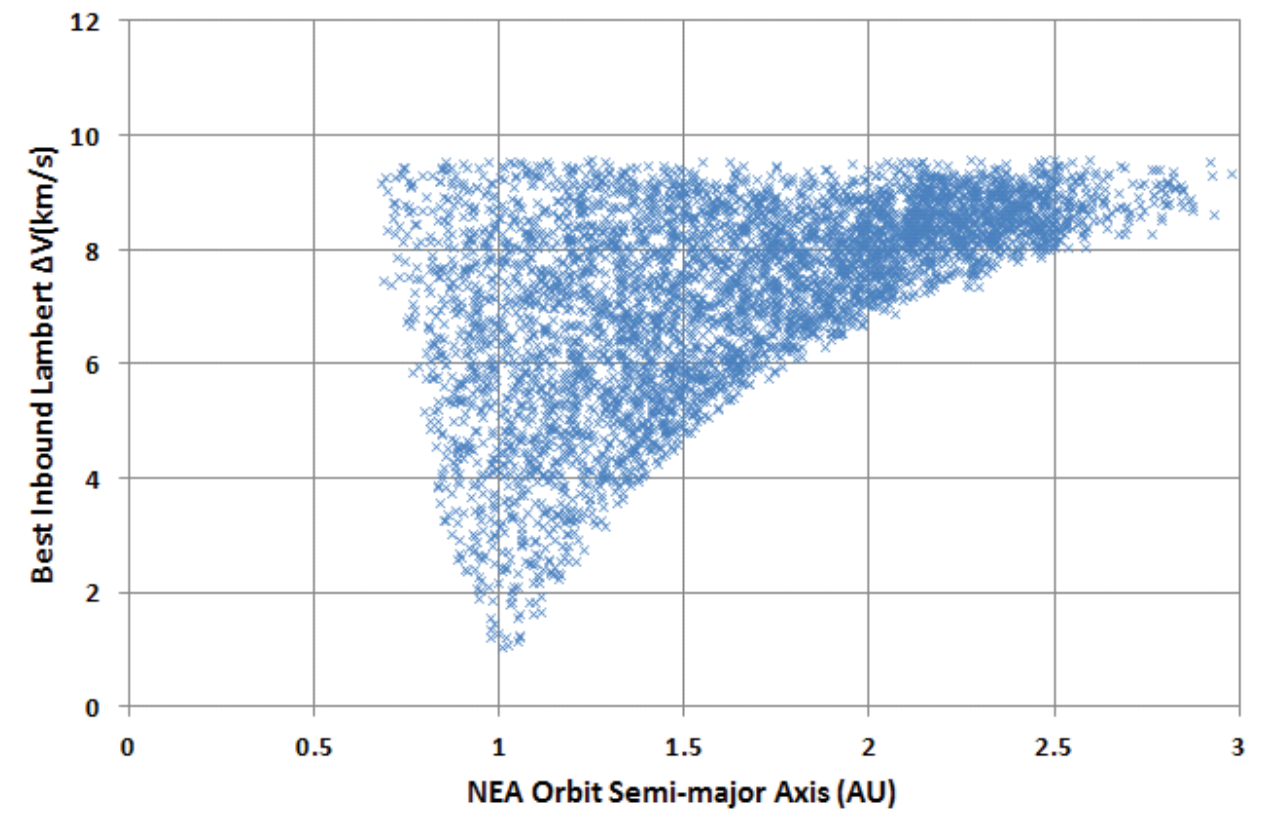

Figure 3. Semi-major axis vs best inbound Lambert $\Delta V$ between 2010 and 2040 for all NEAs recorded

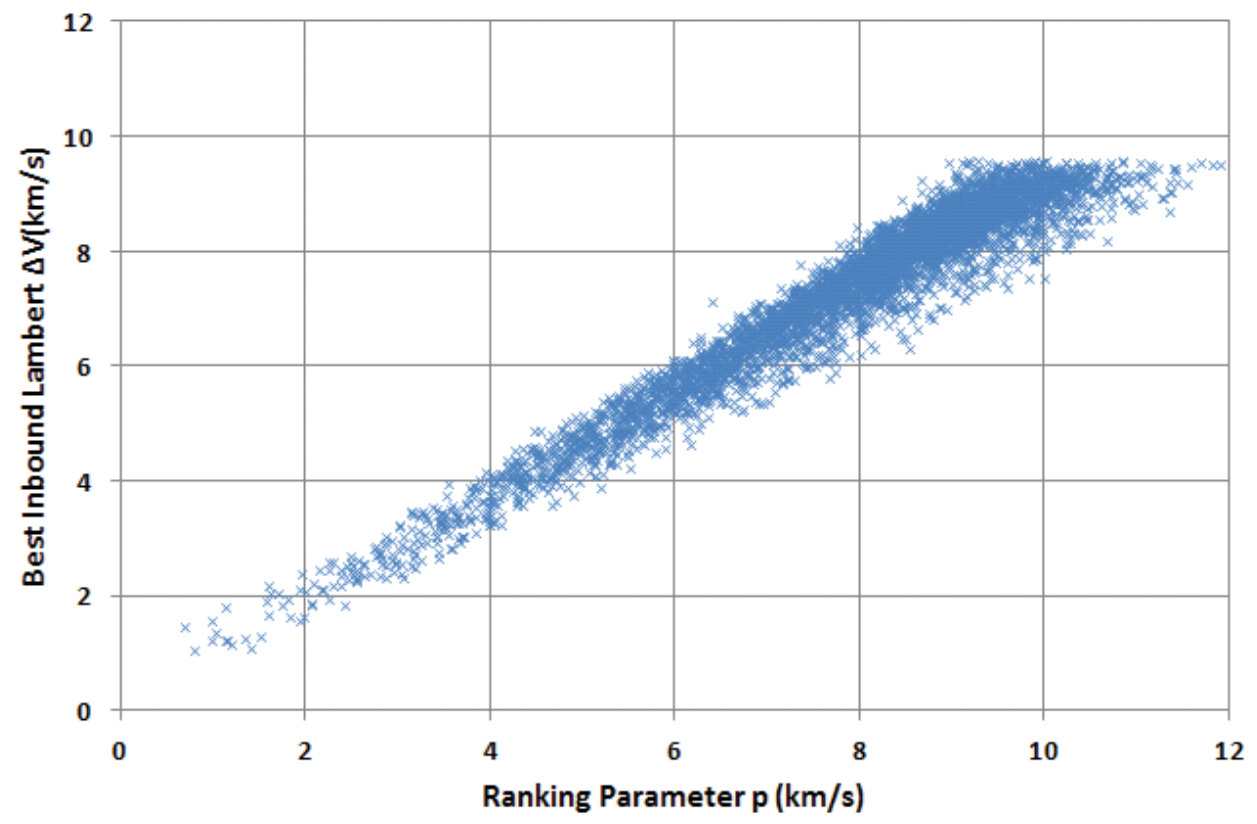

Figure 4. Ranking parameter vs best inbound Lambert $\Delta V$ between 2010 and 2040 for all NEAs recorded

The Lambert scan database can be queried to look at the $\Delta \mathrm{V}$ requirement of a mission to any of the ranked NEAs given the launch year, stay time and return year constraints. Potential mass returned from the NEA can then be estimated from the rocket equation based on the $\Delta V$ requirements, flight time, as well as mass and acceleration constraints of the spacecraft.

Lambert results and any known physical characteristics of the targets are used to identify potential candidates of the ARRM mission. Sizes, types, and the existences of pre-cursor missions are key factors in the selection process. Once a candidate has been identified, mission trajectories are then modeled in mid-fidelity tools such as EMTG and 
MALTO and a series of trade studies are performed to look at potential mass returned using any combination of launch vehicle, engine specific impulse, launch date, stay time and return date.

Power and thrust models were selected based on a level of fidelity appropriate for the low-thrust trajectory modeling of EMTG and MALTO. All trajectories assumed Triple Junction Gallium Arsenide (TJ GaAs) solar cells on a Megaflex or Roll-Out Solar Array (ROSA) array structure capable of generating $51 \mathrm{~kW}$ at the beginning of life at one AU. $500 \mathrm{~W}$ of the power generated was reserved for the spacecraft bus during SEP thrusting and the array power was decayed at $1 \%$ per year. A $15 \%$ power margin was dynamically enforced to conservatively reduce the power available to the electric propulsion system.

Two different Isp levels were evaluated for the electric propulsion (EP) system for all asteroid targets. In thrustconstrained scenarios, a SEP system with an Isp of 2000s at 55\% efficiency can increase the maximum pick-up mass with a higher thrust output for a given power level. A system with an Isp of $3000 \mathrm{~s}$ at $60 \%$ efficiency, however, can produce larger mission $\Delta \mathrm{V}$ at a lower propellant cost if the time of flight allows for the increased periods of thrusting. The maximum power into the EP system for both was $40 \mathrm{~kW}$, and a $90 \%$ duty cycle was enforced to accommodate periods of missed thrusting and thruster misalignment. The Asteroid Robotic Redirect Vehicle (ARRV) would carry multiple thrusters based on the desired Isp, however all trajectories designed in EMTG and MALTO assumed a single mega thruster that produced the equivalent combined thrust and mass flow rate of the multiple thrusters to simplify the modeling. The $2000 \mathrm{~s}$ Isp configuration uses the same magnetically shielded Hall thrusters used in the 3000s Isp configuration, however the input voltage and power are lower. If flown under these assumptions the 2000s Isp configuration will likely carry more thrusters than the $3000 \mathrm{~s}$ configuration, increasing the ARRV dry mass estimate.

For ARRM Option B missions that are launched on a Falcon Heavy or Delta IV Heavy, a pair of lunar gravity assists increases the departure $\mathrm{C} 3$ from $-2 \mathrm{~km}^{2} / \mathrm{s}^{2}$ to $2 \mathrm{~km}^{2} / \mathrm{s}^{2}$. 5,6 Interplanetary trajectory modeling was simplified by assuming a maximum Earth departure mass based on the launch vehicle capability to a $\mathrm{C} 3$ of $-2 \mathrm{~km} \mathrm{~km}^{2} / \mathrm{s}^{2}$, where the upper bound on the departure $\mathrm{C} 3$ was then $2 \mathrm{~km}^{2} / \mathrm{s}^{2}$ with a declination between $+/-30$ degrees. For the Falcon Heavy, the maximum spacecraft mass was assumed to be 13,153 kg for a $2000 \mathrm{~s}$ Isp EP system and 13,169 $\mathrm{kg}$ for a 3,000 s Isp EP system. These values include mass decrements for the propellant assumed to be necessary for any maneuvers to clean up launch vehicle insertion errors and perform phasing before lunar flybys. The estimated ARRV dry mass plus the reaction control system (RCS) propellant limits the available xenon for low-thrust propulsion. The ARRV dry mass including RCS propellant was 5,170 kg for the $2000 \mathrm{~s}$ Isp configuration and 5,020 $\mathrm{kg}$, for the $3000 \mathrm{~s}$ Isp configuration.

Once at the asteroid, the ARRV will conduct proximity operations to characterize the asteroid, extract the boulder, and conduct PD demonstration(s). The time required for characterization is dependent on whether a precursor mission has already been conducted, where less time is required for a characterized asteroid such as Itokawa. The time allocation for boulder acquisition includes a budget for attempting collection 5 total times at up to 3 different locations of the NEA. Time is also budgeted for performing and measuring PD, specifically an enhanced gravity tractor demonstration. This demonstration is dependent on the mass of the asteroid and the mass of the pick-up boulder. The demonstration needs be conducted long enough to perturb the orbital state of the asteroid outside of the navigation uncertainty bounds of the nominal, unaltered orbit and then enough time has to elapse for the perturbation to reach a measurable size For Itokawa, the minimum stay time at the asteroid was determined to be 400 days for a conservative design. This stay time is also used as an estimate for the other asteroids examined with the understanding that the stay would change after a refined proximity operations design for each particular target.

In addition to direct Earth to asteroid outbound legs and asteroid to Earth inbound legs, gravity assist trajectories were also investigated. Global searches for Earth gravity assists on both trajectory legs for non-Mars crossing asteroids was conducted. An Earth flyby on the return leg was frequently beneficial in terms of increasing the maximum boulder pick-up mass except for trajectories that were constrained to have a time of flight shorter than 5 years. For Mars-crossing asteroids, Venus, Earth, Mars flybys were examined to determine whether the return mass could be increased over a direct return.

Finally, integrated end-to-end trajectories were developed in Copernicus, a high-fidelity trajectory modeling tool developed by NASA's Johnson Space Center (JSC), from launch to Earth return to verify assumptions made in theory based analysis. In the mid-fidelity trajectory modeling of EMTG and MALTO, only Sun's gravity is included in the trajectories, and the spacecraft departs from and returns to the center of Earth. In Copernicus, the Sun, Earth Moon's gravities are all included, and the spacecraft departs from low-Earth orbit, performs two lunar flybys before leaving Earth, and upon return, performs additional lunar flybys and goes through a solar perturbation phase before inserting into a Lunar Distant Retrograde Orbit (LDRO). Earth departure and Earth sphere of influence (SOI) arrival dates modeled in Copernicus can be upto 15 days earlier or later than the EMTG and MALTO solutions 
because lunar flybys can only be performed on certain days of the month when the moon is at a favorable location, and the final LDRO insertion date is typically five or six months after the Earth SOI arrival date. However, the return masses, propellant usages obtained from the Copernicus models match very well with the EMTG and MALTO results, which provide further proof that the Earth departure and arrival C3 assumptions used in EMTG and MALTO are well justified.

\section{Target Selection}

Early in the AATS the population of known NEAs were ranked using the ranking parameter defined above, and Lambert scans were run with carefully limited thrust and increased stay time. These two data sets were compared to allow identification of potential targets with large return mass given launch date, desired NEA stay time and return date. These lists were then examined for targets with optical and radar observation opportunites with high enough Visual magnitude (Vmag) and Signal-to-Noise Ration (SNR) for identification of spin rate, shape, spectral type, and surface roughness (a potential indicator of the existence of boulders). While these lists include many targets with acceptable return masses, none of the observation opportunities met the requirements for a potentially selectable target.

While numerous asteroids greater than $100 \mathrm{~m}$ have been discovered via targeted Potentially Hazardous Asteroid (PHA) identification campaigns, only Itokawa (visited by the JAXA spacecraft Hayabusa) and Eros (visited by the NEAR-Shoemaker spacecraft) are currently well-characterized. Itokawa presents an attractive destination for boulder retrieval due to an abundance of boulder options of various dimensions. However, Hayabusa is not the only precursor mission that can be leveraged to ensure attractive boulder options exist. The OSIRIS-Rex spacecraft will visit Bennu in 2018 and the Hayabusa 2 spacecraft will also rendezvous with its target asteroid (1999 JU3) in 2018. Additionally, the asteroid 2008 EV5 has been relatively well characterized via radar (SNR > 5000). These four potential asteroid targets represent the prime options for Option B, but are not the only possible targets. Even though a premium has been placed on targets that are potentially carbonaceous, any asteroid with a high-quality radar and visual observation opportunity in the near future represents a potentially viable target assuming the minimum feasible pick-up boulder size is approximately $2 \mathrm{~m}$ maximum extent. As NEAs are detected that are potential Option B targets, observation assets are being used to gather as much information about each target as possible. Several targets with potential large return mass have been detected including NEA $2014 \mathrm{EK}_{24}$, however its spectral color type is consistent with $\mathrm{S}$ or $\mathrm{V}$ type and its rotation period is only 6 minutes ${ }^{17}$ making it not an attractive ARRM AATS target.

\section{Results}

MALTO and EMTG trajectory analysis for the 4 potential ARRM Option B targets was run for 3 potential launch vehicles with launch no earlier than June 1 (heliocentric Earth departure no earlier than September 27) in 2019, 2020, and 2021, based on specific programmatic constraints. Return of the spacecraft and boulder to a stable LDRO (6 months after initial ECA or LGA on return) was constrained to $2024-2029$ where the optimal return date for each year was chosen. This wide range of mission dates and launch vehicles identifies the implications of programmatic decisions made on required launch and return dates. Early in the study it became clear that the size of a boulder's maximum extent rather than the mass of the returned boulder was the metric being used by decision makers to determine if the returned boulder was sufficiently large. A conservative approximation was used for size based on predicted density derived from visited asteroids and the meteorite collections $\left(3.22 \mathrm{~g} / \mathrm{cm}^{3}\right.$ for stony and $1.62 \mathrm{~g} / \mathrm{cm}^{3}$ for carbonaceous) and a worst case maximum extent assuming a spherical boulder. Table 1 includes both the mass in tonnes and diameter of the largest boulder that can be returned with or without an Earth gravit assist on the inbound leg for a mission with a 400 day stay at the NEA.

An Earth gravity assist on the return leg frequently increased the maximum return mass, particularly for missions with at least 5 year time of flight (total mission duration of 6 years). The Earth gravity assist is especially beneficial for asteroids with relatively high inclinations, where the Earth flyby can effectively enable a propellant-free plane change. In the case of boulder return from $2008 \mathrm{EV}_{5}$, with an inclination of 7.4 degrees from the ecliptic, an Earth gravity assist improved the maximum boulder return mass by up to $80 \%$ for mission durations greater than 6 years (E.g. $2019-2025)$. 
Table 1. Maximum boulder pick-up mass in metric tons for Itokawa, Bennu, $1999 \mathrm{JU}_{3}$, and $2008 \mathrm{EV}_{5}$ with a Falcon Heavy, Delta IV Heavy, or SLS 1B launch vehicle
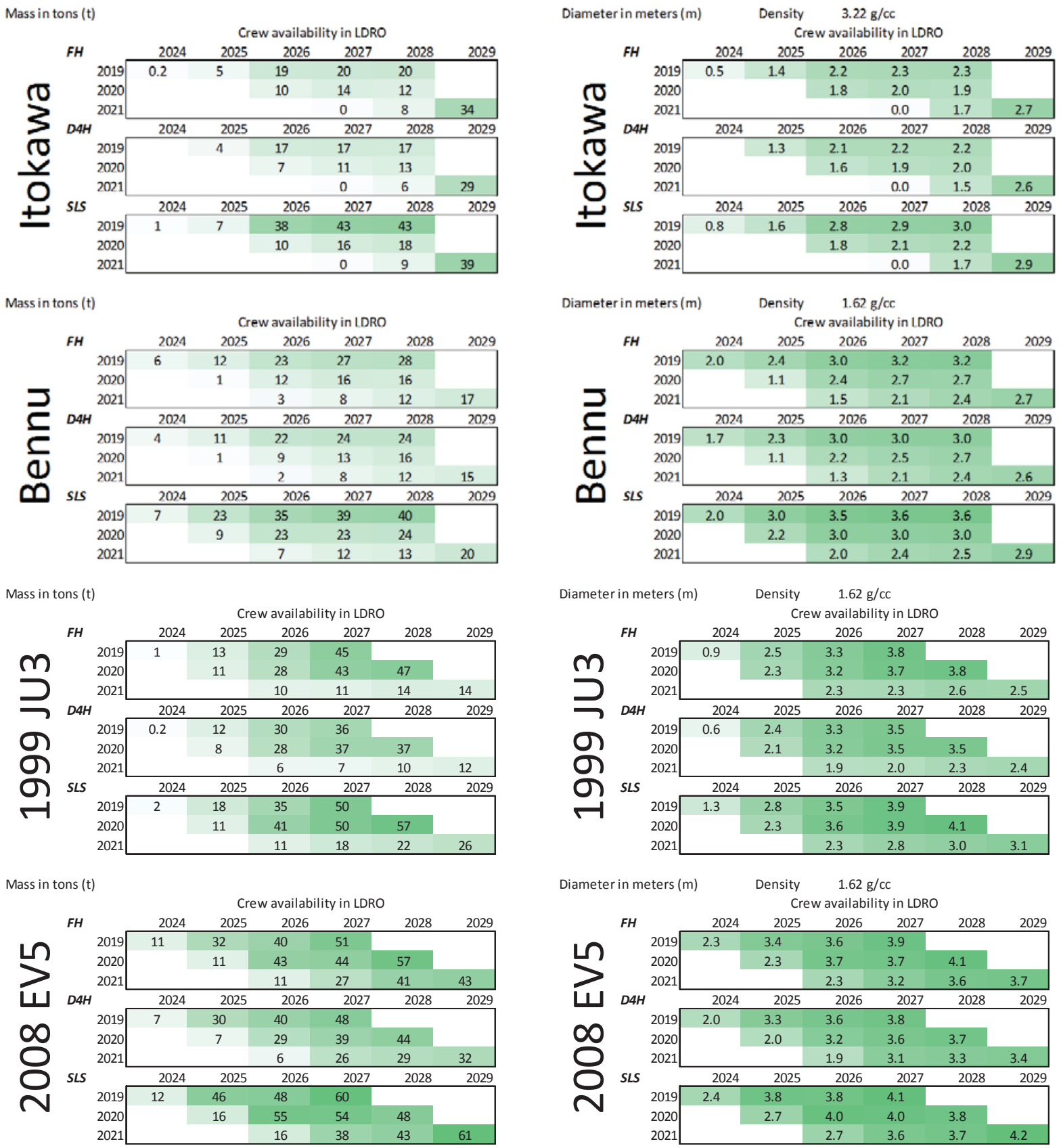

NASA Headquarters specified that the boulder must be in the LDRO for crew accessibility in 2024 to align with an early SLS and Orion crew mission. In order to increase the size of the boulder returned for missions with short total duration NEA stay time was reduced from 400 days to 120 days with the understanding that planetary defense will be demonstrated, but will not necessarily cause an observable change the the NEAs orbit.

A heliocentric plot of the Bennu (1999 RQ $\left.{ }_{36}\right)$ MALTO case with this updated stay time assumptions with an SLS cargo launch vehicle in 2019 is shown in Figure 5. 


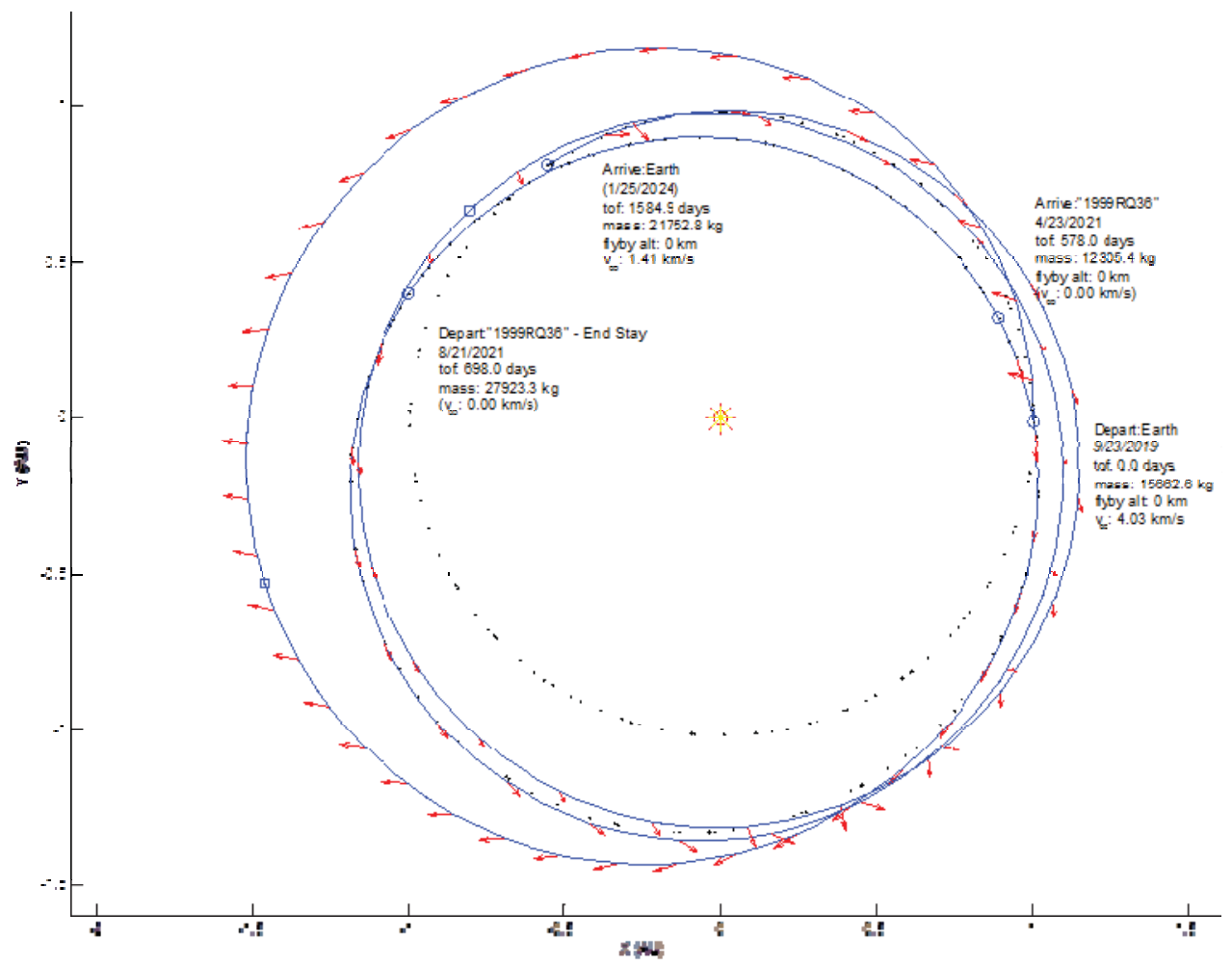

Figure 5. MALTO trajectory for Bennu 2019 Launch on an SLS with 2024 crew availability

For comparison the heliocentric trajectory from an end-to-end integrated Copernicus trajectory is shown in Figure 6. Note that the Earth departure dates are identical, because phasing for an LGA is not necessary with the direct trans NEA injection of the SLS.
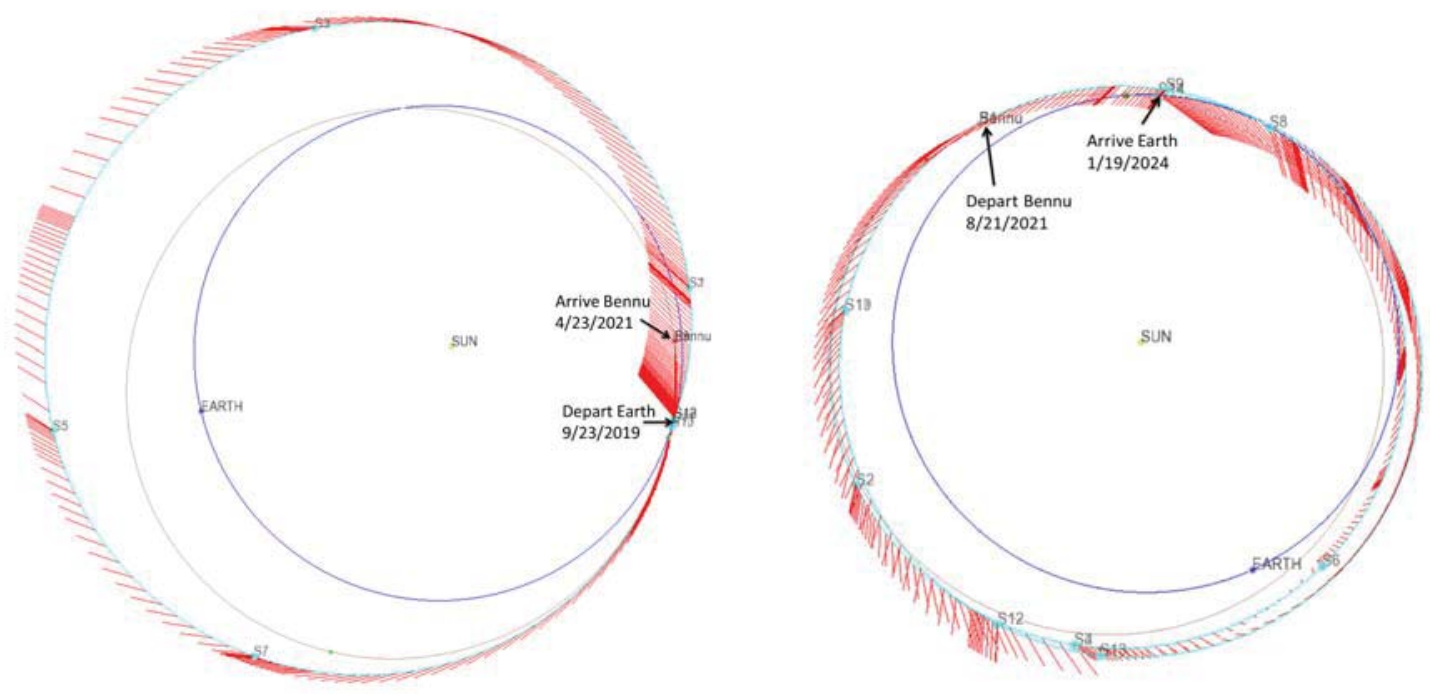

Figure 6. Copernicus trajectory for Bennu 2019 Launch on an SLS with 2024 crew availability 
It was found that a 3-burn departure sequence allows departure at the optimal departure declination after a due east launch from the Kennedy Space Center. The first burn raises the apogee, the second burn near apogee changes the orbit's plane to align for departure, and the third burn at perigee (trans NEA injection) completes SLS maneuvers approximately 5 days after launch. In both trajectories Bennu arrival and departure dates are also identical; however the Earth sphere of infleuence arrival had to be phased in Copernicus to allow a pair of LGAs half a month apart for alignment of a solar perturbation loop and finally capture into the LDRO. Figure 7 shows these maneuvers for the 1/19/2024 Earth arrival from Bennu in an Earth centered J2000 frame.

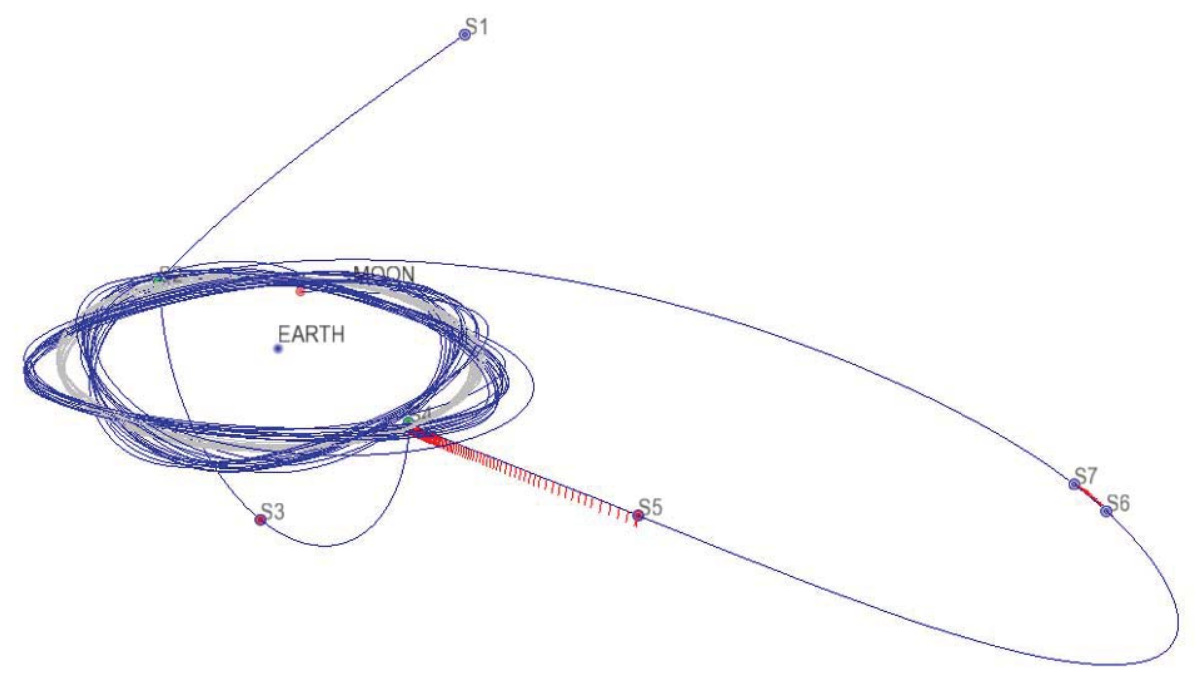

Figure 7. ARRM Earth capture and LDRO insertion

The predicted return mass is lower by approximately $800 \mathrm{~kg}$ or $0.5 \%$ in the integrated trajectory developed in Copernicus. Table 2 is comparison of key parameters from the MALTO and Copernicus trajectories for this Bennu trajectory. This difference is due to modifying the return leg to allow for LGA capture. For mission options that use the Falcon Heavy or the Delta IV Heavy both the Earth depature and arrival include LGAs and must be phased accordingly by moving the departure or arrival date by up to 15 days for correct lunar alignment.

Table 2. Key date and Mass comparison for MALTO and Copernicus trajectory

\begin{tabular}{|l|r|r|r|r|}
\hline & \multicolumn{2}{|c|}{ MALTO } & \multicolumn{2}{c|}{ Copernicus } \\
\hline Event & \multicolumn{1}{|c|}{ Date } & \multicolumn{1}{c|}{ Mass } & \multicolumn{1}{c|}{ Date } & \multicolumn{1}{c|}{ Mass } \\
\hline Earth Departure & $9 / 23 / 2019$ & 15662.6 & $9 / 23 / 2019$ & 15511.8 \\
\hline NEA Arrival & $4 / 23 / 2021$ & 12305.4 & $4 / 23 / 2021$ & 12189.9 \\
\hline NEA Departure & $8 / 21 / 2021$ & 27923.3 & $8 / 21 / 2021$ & 27000 \\
\hline Earth SOI arrival & $1 / 25 / 2024$ & 21752.76 & $1 / 19 / 2024$ & 21393.4 \\
\hline LDRO insertion & & & $7 / 5 / 2024$ & 21342 \\
\hline Boulder Mass & & 16017.9 & & 15210.1 \\
\hline
\end{tabular}


The distance from the spacecraft to the Sun (Figure 7) is critical to mission performance since the amount of power generated by the solar arrays drives the performance of the electric propulsion system. Spacecraft thermal performance constrains the trajectory to solar distances greater than $0.8 \mathrm{AU}$; in this trajectory the minimum spacecraft distance to the sun is approximately 0.9 AU. Distance from the spacecraft to Earth is also calculated and used for communications planning (Figure 7). Due to the phasing of Bennu and the Earth the distance between the two is large ranging from 1.9 AU to 2.1 AU while at Bennu.

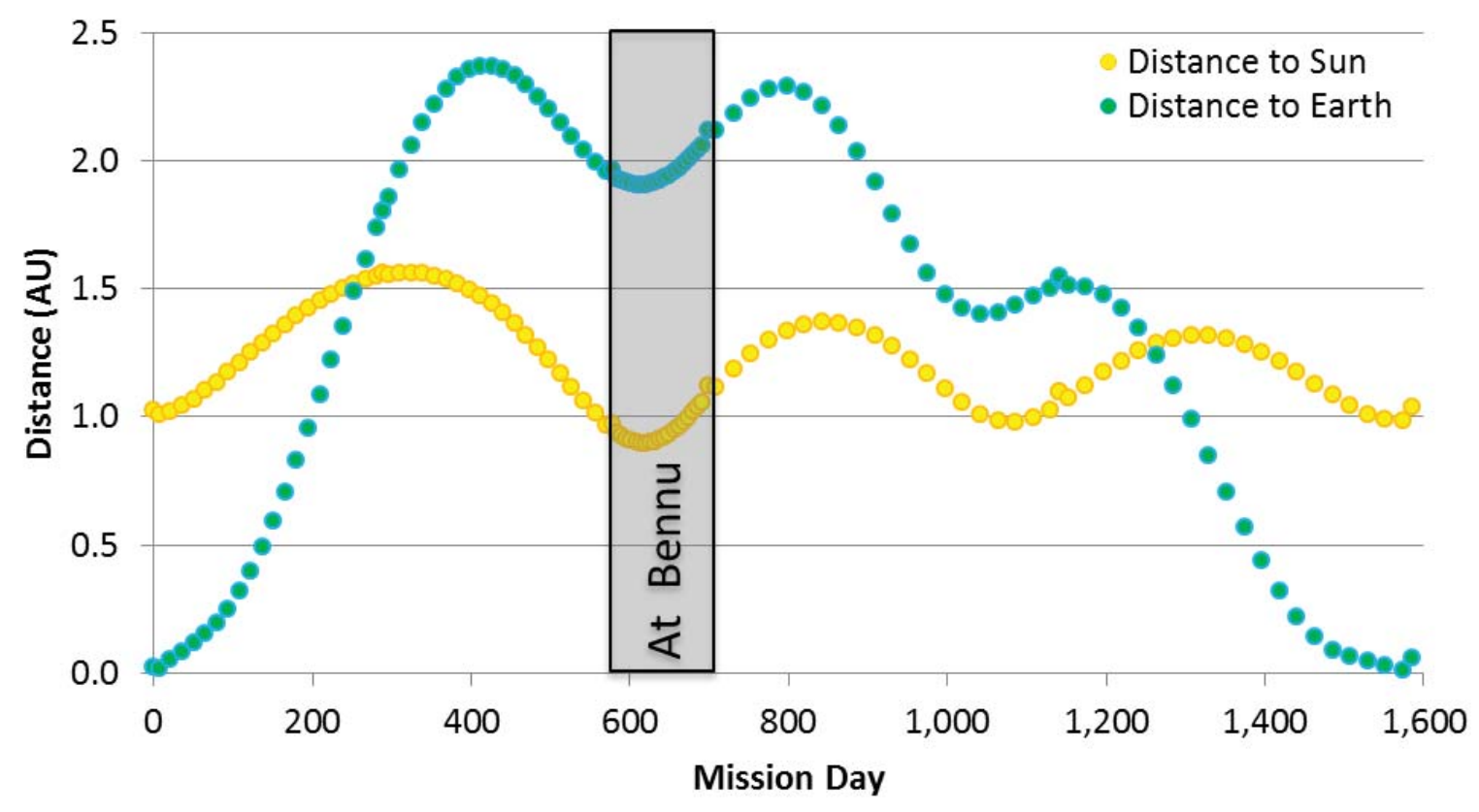

Figure 7. Spacecraft distance to Sun and Earth for Bennu 2019 Launch on an SLS with 2024 crew
availability

As the AATS progressed assessment of the earliest possible return dates was requested for candidate NEAs. EMTG was used to develop return performance as a function of time data sets. Figure 8 and Figure 9 are early return performance for Bennu and $2008 \mathrm{EV}_{5}$. A mission to Bennu with a $5 \mathrm{t}$ return (2 meter spherical extent) returns as early as January 2024 with an SLS launch vehicle and a SEP I $\mathrm{I}_{\mathrm{sp}}$ of 2000s. A similar $2008 \mathrm{EV}_{5}$ mission can return in January 2023. If the stay time is extended from 120 days to 400 days the return dates slip to May 2024 for Bennu and July 2023 for $2008 \mathrm{EV}_{5}$. Moving to a smaller launch vehicle like the Falcon Heavy only slips the return date for Bennu by one or two months for $\sim 2$ meter boulders, however the $2008 \mathrm{EV}_{5}$ retuns slip by 3 or 4 months. 


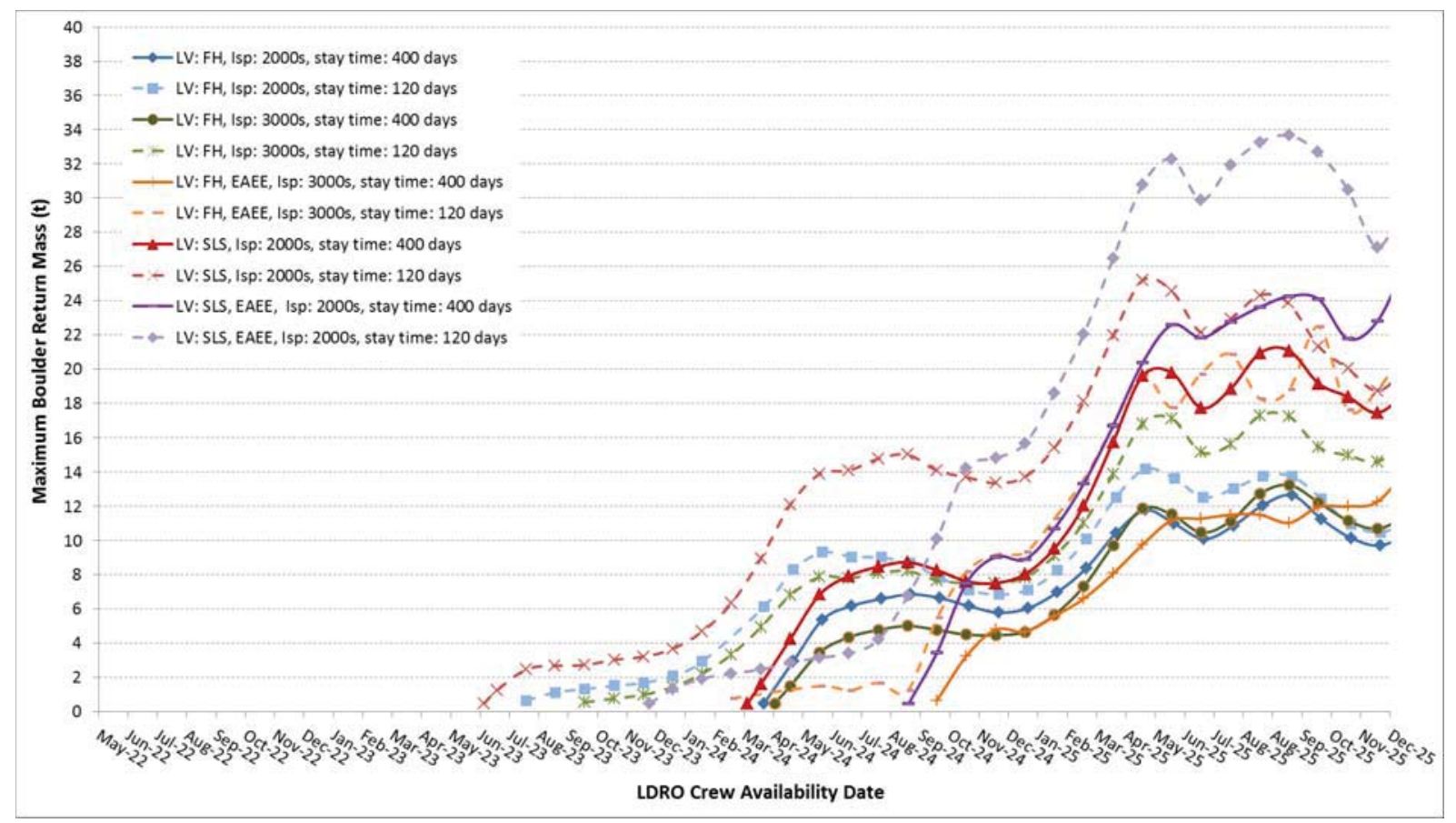

Figure 8. Bennu Early Return Performace

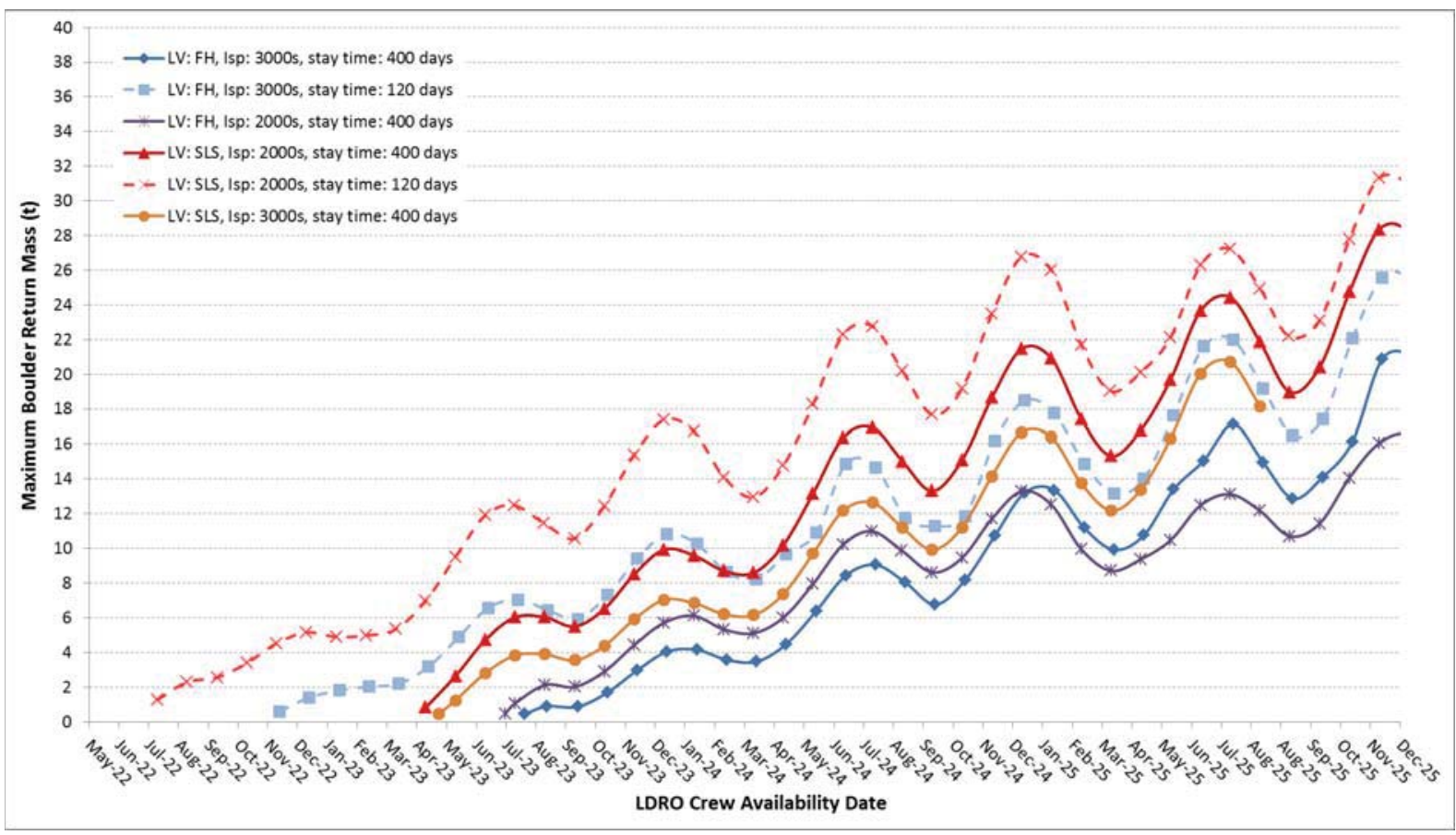

Figure 9. 2008 EV $_{5}$ Early Return Performace 


\section{Conclusion}

Four NEAs have been identified that meet the selection criteria for ARRM Option B and permit return of boulders to LDRO for crew availability in 2024. With proper selection of trajectory and spacecraft performance characteristics, it is expected that a mission to any of these targets can fulfill NASA's goal of sending crew to asteroidal material in the mid 2020s. The target selection and trajectory analysis process detailed above permits both a rapid survey of many candidate targets, as well as accurate characterization of potential return mass. This approach permits the consideration of both direct and gravity assist trajectories to trade mission duration and return mass. As new NEAs are discovered, methods discussed in this paper are applied to determine potential boulder return mass. Several have been found in 2014 with large potential return mass, however none have been observed that meet the selection critera for ARRM Option B.

Since completion of the AATS both ARRM Option A and Option B concepts are being matured and risk reduction activites are ongoing into the fall of 2014. A downselect to one option is planned for late calendar year 2014 with a Mission Concept Review (MCR) in early 2015.

\section{Acknowledgments}

The authors thank Lindley Johnson and John Grunsfeld for making the AATS possible and Dan Mazanek, the ARRM Option B lead, for his guidance.

\section{References}

${ }^{1}$ J. Brophy et al, “Asteroid Retrieval Feasibility Study,” Keck Institute for Space Studies Report, April 2012.

${ }^{2}$ N. Strange, D. Landau, T. McElrath, G. Lantoine, T. Lam, M. McGuire, L. Burke, M. Martini, J. Dankanich, "Overview of Mission Design for NASA Asteroid Redirect Robotic Mission Concept," presented at the $33^{\text {rd }}$ International Electric Propulsion Conference, The George Washington University, Washington, D.C., October 2013.

${ }^{3}$ D. Mazanek, J. Brophy, G. Merrill, "Asteroid Retrieval Mission Concept - Trailblazing Our Future in Space and Helping to Protect Us from Earth Impactors," $3^{\text {rd }}$ IAA Planetary Defense Conference, Flagstaff, AZ April 2013.

${ }^{4}$ Busch et al., Radar Observations and the Shape of Near-Earth Asteroid 2008 EV5", Icarus Volume 212, Issue 2, April 2011, Pages 649-660

${ }^{5}$ Personal Communications with Michael Busch

${ }^{6}$ D. Landau, J. Dankanich, N. Strange, J. Bellerose, P. Llanos, M. Tantardini, "Trajectories to Nab a NEA (Near Earth Asteroid)", Paper AAS 13-409, 2013.

${ }^{7}$ D. Landau, T. McElrath, D. Grebow, N. Strange, "Efficient Lunar Gravity Assists for Solar Electric Propulsion Missions," Paper AAS 12-165, Feb. 2012.

${ }^{8}$ T. McElrath, et al., "Using Gravity Assists in the Earth-Moon System as a Gateway to the Solar System," Paper GLEX 2012.05.5.2x12358, May 2012.

${ }^{9}$ J. Englander and E. Cardiff, “Asteroid Retrieval via Direct Launch and Solar Electric Propulsion,” AAS/AIAA Space Flight Mechanics Meeting, Santa Fe, NM, February 2014.

${ }^{10}$ J. Englander, B. Conway, and T. Williams, "Automated Mission Planning via Evolutionary Algorithms," Journal of Guidance, Control, and Dynamics, Vol. 35, No. 6, 2012, pp. 1878-1887.

${ }^{11}$ J. A. Englander, B. A. Conway, and T. Williams, "Automated Interplanetary Mission Planning," AAS/AIAA Astrodynamics Specialist Conference, Minneapolis, MN, August 2012.

${ }^{12}$ D. H. Ellison, J. A. Englander, and B. A. Conway, "Robust Global Optimization of Low-Thrust, Multiple-Flyby Trajectories," AAS/AIAA Astrodynamics Specialist Conference, Hilton Head, SC, August 2013.

${ }^{13}$ J. Englander, D. Ellison, and B. Conway, "Global Optimization of Low-Thrust, Multiple-Flyby Trajectories at Medium and Medium-High Fidelity,” AAS/AIAA Space Flight Mechanics Meeting, Santa Fe, NM, February 2014.

${ }^{14}$ D. H. Ellison, J. A. Englander, M. T. Ozimek, and B. A. Conway, "Analytical Partial Derivative Calculation of the SimsFlanagan Transcription Match Point Constraints," AAS/AIAA Space-Flight Mechanics Meeting, Santa Fe, NM, January 2014.

${ }^{15}$ J. Sims, P. Finlayson, E. Rinderle, M. Vavrina, and T. Kowalkowski, "Implementation of a low-thrust trajectory optimization algorithm for preliminary design," AIAA/AAS Astrodynamics Specialist Conference, August 2006.

${ }^{16}$ C. Ocampo, J. Senent, "The Design and Development of COPERNICUS: A Comprehensive Trajectory Design and Optimization System”, Paper IAC-06-C1.4.04, Oct. 2006.

${ }^{17}$ Personal Communications with Paul Abell 